\title{
Simple models for strictly non-ergodic stochastic processes of macroscopic systems
}

\author{
G. George, L. Klochko, A.N. Semenov, J. Baschnagel, and J.P. Wittmer \\ Institut Charles Sadron, Université de Strasbourg \& CNRS, 23 rue du Loess, 67034 Strasbourg Cedex, France
}

Received: date / Revised version: date

\begin{abstract}
We investigate simple models for strictly non-ergodic stochastic processes $x_{t}$ ( $t$ being the discrete time step) focusing on the expectation value $v$ and the standard deviation $\delta v$ of the empirical variance $v[\mathbf{x}]$ of finite time series $\mathbf{x} . x_{t}$ is averaged over a fluctuating field $\sigma_{\mathbf{r}}$ ( $\mathbf{r}$ being the microcell position) characterized by a quenched spatially correlated Gaussian field $g_{\mathbf{r}}$. Due to the quenched $g_{\mathbf{r}}$-field $\delta v(\Delta \tau)$ becomes a finite constant, $\Delta_{\text {ne }}>0$, for large sampling times $\Delta \tau$. The volume dependence of the non-ergodicity parameter $\Delta_{\text {ne }}$ is investigated for different spatial correlations. Models with marginally long-ranged $g_{\mathbf{r}}$-correlations are successfully mapped on shear-stress data from simulated amorphous glasses of polydisperse beads.
\end{abstract}

\section{Introduction}

It is common to characterize a stochastic process $x(\tau)[1$ using ensembles $\{\mathbf{x}\}$ of discrete time series

$$
\mathbf{x}=\left\{x_{t}=x\left(\tau_{t}=t \delta \tau\right), t=1, \ldots, n_{\mathrm{t}}\right\}
$$

with $\tau$ being the continuous time, $t$ the discrete time, $\delta \tau$ the time interval between the equidistant measurements and $\Delta \tau=n_{\mathrm{t}} \delta \tau$ the experimentally or computationally available "sampling time" [2, 3, 4, 5, 6. Let us denote by $\mathcal{O}[\mathbf{x}]$ a functional of a given time series $\mathbf{x}$. If the stochastic process $x(\tau)$ is ergodic [7, the expectation value $\mathcal{O}$ and the standard deviation $\delta \mathcal{O}$ of $\mathcal{O}[\mathbf{x}]$ may be obtained by either averaging over ensembles $\left\{\mathbf{x}_{c}, c=1, \ldots, n_{\mathrm{c}}\right\}$ of independent "configurations" $c$ ("c-averaging") or over ensembles $\left\{\mathbf{x}_{k}, k=1, \ldots, n_{\mathrm{k}}\right\}$ of time series $k$ of one large trajectory $c$ (" $k$-averaging") exploring a significant representative part of the generalized phase space of the system. It is thus sufficient for such ergodic systems to characterize the time series $\mathbf{x}$ by one index $c$ or $k \bigsqcup^{1}$

The ergodicity hypothesis is in fact violated in many physical, biological and socio-economic systems, i.e. even very long " $c$-trajectories" remain trapped (at least in practice) in "meta-basins" of a generalized phase space [1, 5, 6 7,8, 9. (For Hamiltonian dynamical systems such basins correspond simply to valleys of the potential energy landscape [8], for more general stochastical dynamical schemes to valleys of the relevant free energy landscape quantified by the minimal external work needed to quasistatically push the system into a specific state point.) Modelling the statistics and dynamics of such non-ergodic processes has become of paramount importance, especially in conjunction with advanced experimental techniques, such as

a joachim.wittmer@ics-cnrs.unistra.fr

1 We assume $n_{\mathrm{c}} \gg 1$ and $n_{\mathrm{k}} \gg 1$ throughout this work. single particle tracing in cells [10. Importantly, a time series $\mathbf{x}_{c k}$ must now be characterized by two indices $c$ and $k$ and it becomes crucial in which order $c$ - and $k$-averages are taken [6]. As a consequence, the standard total variance

$$
\delta \mathcal{O}_{\text {tot }}^{2}(\Delta \tau)=\delta \mathcal{O}_{\text {int }}^{2}(\Delta \tau)+\delta \mathcal{O}_{\text {ext }}^{2}(\Delta \tau)
$$

is the sum of two contributions characterizing, respectively, the internal variance within each $c$ and the external variance between different $c$. Moreover, for large sampling times $\delta \mathcal{O}_{\text {int }} \rightarrow 0$ while $\delta \mathcal{O}(\Delta \tau) \simeq \delta \mathcal{O}_{\text {ext }}(\Delta \tau)$ approaches for non-ergodic systems a positive definite constant $\Delta_{\text {ne }} \equiv$ $\lim _{\Delta \tau \rightarrow \infty} \delta \mathcal{O}_{\text {ext }}(\Delta \tau)$. This is the relevant "non-ergodicity parameter" [5, 6] of this study. (See Sec. 2.2 for more details.) Fortunately, $\Delta_{\text {ne }}$ decreases generally with the system volume $V$ for processes with a large number $n_{\mathbf{r}} \propto V$ of more or less independent microcells $[5]$.

One goal of the present work is to introduce some useful operator notations allowing to characterize concisely fluctuations of ensembles of non-ergodic systems and to illustrate the above statements by means of various simple stochastic models which can be treated (essentially) analytically. Moreover, we attempt to describe the system-size dependence of $\Delta_{\text {ne }}$ by means of two-point spatial correlation functions of an effective quenched microscopic field $g_{\mathbf{r}}$ related to the $k$-averaged standard deviation $s_{\mathbf{r}}$ of a microscopic fluctuating field $\sigma_{\mathbf{r}}$ ( $\mathbf{r}$ labeling the microcell position). As in our recent studies [3, 4, 5, 6] we focus on the empirical variance $v[\mathbf{x}]$ (defined in Sec. 2.3) and the corresponding expectation value $v(\Delta \tau)$ and the standard deviations $\delta v_{\text {int }}(\Delta \tau)$ and $\delta v_{\text {ext }}(\Delta \tau)$. One important motivation is that many physical quantities can be obtained by equilibrium molecular dynamics (MD) or Monte Carlo (MC) simulations [11,12 using fluctuation dissipation relations [7,11, 13, 14, 15, 16]. Understanding how the respective variances and their standard deviations depend on the 
length $\Delta \tau$ of the production runs and the simulation box volume $V$ is thus crucial $3,4,5,6,16$.

We recall first in Sec. 2 recent results [4,5,6] and discuss then in Sec. 3 the $V$-dependence of various properties and, more specifically, how $\Delta_{\text {ne }}(V)$ may depend on spatial correlations (Sec. 3.3) under the physically motivated constraint that the expectation value of the variance $v$ must be $V$-independent (Sec. 3.2). We turn then in Sec. 4 to the description of different imposed $g_{\mathbf{r}}$-distributions (Sec. 4.2 . Model variants are mapped in Sec. 5 onto simulated data obtained from the shear stresses in amorphous glasses 2, 2, 5,6. Our results are summarized in Sec. 6. The numerical generation of spatially correlated Gaussian fields is discussed in Appendix $\mathrm{A}$ and an alternative quenched field important for future work 17. in Appendix B.

\section{Makroscopic properties}

\subsection{Some useful notations}

It is useful to introduce a few notations. The $l$-average operator

$$
\mathbf{E}^{l} \mathcal{O}_{l m n \ldots} \equiv \frac{1}{n_{l}} \sum_{l=1}^{n_{l}} \mathcal{O}_{l m n \ldots} \equiv \mathcal{O}_{m n \ldots}\left(n_{l}\right)
$$

takes a property $\mathcal{O}_{l m n \ldots}$ depending possibly on several indices $l, m, \ldots$ and projects out the specified index $l$, i.e. the $l$-average $\mathcal{O}_{m n \ldots}\left(n_{l}\right)$ does not depend any more on $l$, but it may depend on the upper bound $n_{l}$ as marked by the argument. Introducing the power-law operator $\mathbf{P}^{\alpha} \mathcal{O} \equiv \mathcal{O}^{\alpha}$, with the exponent $\alpha=2$ being here the only relevant case, and using the standard commutator $[\mathbf{A}, \mathbf{B}] \equiv \mathbf{A B}-\mathbf{B A}$ for two operators $\mathbf{A}$ and $\mathbf{B}$, the $l$-variance operator is defined by $\mathbf{V}^{l} \equiv\left[\mathbf{E}^{l}, \mathbf{P}^{2}\right]$. Note that the $l$-variance

$$
\delta \mathcal{O}_{m n \ldots}^{2}\left(n_{l}\right) \equiv \mathbf{V}^{l} \mathcal{O}_{l m n \ldots}
$$

depends as well in general on the upper bound $n_{l}$. For many cases considered below $\mathcal{O}_{l m n \ldots}\left(n_{l}\right)$ and $\delta \mathcal{O}_{l m n \ldots}\left(n_{l}\right)$ converge for large $n_{l}$ (formally $n_{l} \rightarrow \infty$ ) or become stationary for the experimentally and numerically accessible $n_{l}$-range. This limit is denoted by $\mathcal{O}_{m n \ldots}$ and $\delta \mathcal{O}_{l m n \ldots}$ without the argument $n_{l}$. As discussed in detail in Ref. [6], we have defined $\mathbf{V}^{l}$ as an uncorrected (biased) sample variance operator without the standard Bessel correction [12], i.e. we normalize with $1 / n_{l}$ and not with $1 /\left(n_{l}-1\right)$. This difference is irrelevant for all cases with $n_{l} \gg 1$.

\subsection{Extended $c k$-ensemble for non-ergodic systems}

As stated in the Introduction, for non-ergodic systems a time series $\mathbf{x}_{c k}$ must be characterized by two discrete indices $c$ and $k$ with $c$ standing for the independently generated configuration and $k$ for a subset of length $n_{\mathrm{t}}$ of a much larger trajectory generated for a fixed configuration $c$. Importantly, the $k$-averages

$$
\begin{aligned}
\mathcal{O}_{c}\left(\Delta \tau, n_{\mathrm{k}}\right) & \equiv \mathbf{E}^{k} \mathcal{O}\left[\mathbf{x}_{c k}\right] \text { and } \\
\delta \mathcal{O}_{c}^{2}\left(\Delta \tau, n_{\mathrm{k}}\right) & \equiv \mathbf{V}^{k} \mathcal{O}\left[\mathbf{x}_{c k}\right]
\end{aligned}
$$

depend in general not only on the sampling time $\Delta \tau=$ $n_{\mathrm{t}} \delta \tau$ and the number $n_{\mathrm{k}}$ of time series probed but also on $c$ (as marked by the index) $2^{2}$ The three types of variances mentioned in Sec. 1 are defined by

$$
\begin{aligned}
\delta \mathcal{O}_{\text {tot }}^{2}(\Delta \tau) & \equiv\left[\mathbf{E}^{c} \mathbf{E}^{k}, \mathbf{P}^{2}\right] \mathcal{O}\left[\mathbf{x}_{c k}\right] \\
\delta \mathcal{O}_{\text {int }}^{2}(\Delta \tau) & \equiv \mathbf{E}^{c} \delta \mathcal{O}_{c}^{2}(\Delta \tau)=\mathbf{E}^{c} \mathbf{V}^{k} \mathcal{O}\left[\mathbf{x}_{c k}\right] \\
\delta \mathcal{O}_{\text {ext }}^{2}(\Delta \tau) & \equiv \mathbf{V}^{c} \mathcal{O}_{c}(\Delta \tau)=\mathbf{V}^{c} \mathbf{E}^{k} \mathcal{O}\left[\mathbf{x}_{c k}\right]
\end{aligned}
$$

Using the identity $\left[\mathbf{E}^{c} \mathbf{E}^{k}, \mathbf{P}^{2}\right]=\mathbf{E}^{c} \mathbf{V}^{k}+\mathbf{V}^{c} \mathbf{E}^{k}[6$, it is seen that Eq. (2) exactly holds. The dependencies of the variances on $\Delta \tau, n_{\mathrm{c}}$ and $n_{\mathrm{k}}$ are discussed in detail in Ref. 6]. Importantly, the expectation value of $\delta \mathcal{O}_{\text {tot }}(\Delta \tau)$ for $n_{\mathrm{c}} \rightarrow \infty$ is strictly $n_{\mathrm{k}}$-independent and may also be computed using $n_{\mathrm{k}}=1 . \delta \mathcal{O}_{\text {tot }}^{2}(\Delta \tau)$ is thus the standard commonly computed variance [16, 3, 4, 5]. The "internal variance" $\delta \mathcal{O}_{\text {int }}^{2}(\Delta \tau)$ and the "external variance" $\delta \mathcal{O}_{\text {ext }}^{2}(\Delta \tau)$ depend on $n_{\mathrm{k}}$ in principle, however, for $n_{\mathrm{k}} \gg 10$ the $n_{\mathrm{k}^{-}}$ dependence is only relevant for ergodic systems for which $\delta \mathcal{O}_{\text {ext }} \propto 1 / \sqrt{n_{\mathrm{k}}}[6$ ] and not for the strictly non-ergodic systems we focus on in the present work. For sampling times $\Delta \tau$ much larger than the typical relaxation time $\tau_{\mathrm{b}}$ of the basins we have quite generally

$$
\left.\begin{array}{l}
\delta \mathcal{O}_{\text {int }}(\Delta \tau) \simeq \sqrt{\tau_{\mathrm{b}} / \Delta \tau} \\
\delta \mathcal{O}_{\text {ext }}(\Delta \tau) \simeq \Delta_{\text {ne }}
\end{array}\right\} \text { for } \Delta \tau \gg \tau_{\mathrm{b}}
$$

with the "non-ergodicity parameter" $\Delta_{\text {ne }}$ defined by

$$
\Delta_{\mathrm{ne}} \equiv \lim _{\Delta \tau \rightarrow \infty} \delta \mathcal{O}_{\mathrm{ext}}(\Delta \tau) \equiv \delta \mathcal{O}_{\mathrm{ext}}
$$

Note that $\Delta_{\text {ne }}>0$ only holds for strictly non-ergodic systems while $\Delta_{\mathrm{ne}}=0$ for finite $\tau_{\alpha}[6]$. The first asymptotic law in Eq. 10 is due to the $\Delta \tau / \tau_{\mathrm{b}}$ uncorrelated subintervals for each $c$-trajectory while the second limit is a consequence of the $\mathcal{O}_{c}(\Delta \tau)$ becoming constant. Equation 10 implies

$$
\delta \mathcal{O}_{\text {tot }}(\Delta \tau) \rightarrow \Delta_{\text {ne }} \text { for } \Delta \tau \gg \tau_{\text {ne }} \gg \tau_{\mathrm{b}}
$$

where the crossover time $\tau_{\text {ne }}$ to the $\Delta_{\text {ne}}$-dominated regime is given by $\delta \mathcal{O}_{\text {int }}\left(\tau_{\text {ne }}\right)=\Delta_{\text {ne }}[6$. The numerical importance of the inequality $\tau_{\text {ne }} \gg \tau_{\mathrm{b}}$ is emphasized below.

\subsection{Stationarity}

We assume that each $c$-trajectory in its basin is a stationary stochastic process whose joint probability distribution does not change when shifted in time [1]. This may always be achieved by tempering the system over a tempering time $\tau_{\text {temp }} \gg \tau_{\mathrm{b}}$. To take advantage of the stationarity condition we need to introduce several additional properties. Let us begin by defining the "empirical sample variance" $v[\mathbf{x}] \equiv \mathbf{V}^{t} x_{t}$ of a time series $\mathbf{x}$. By taking the $k$ average $v_{c} \equiv \mathbf{E}^{k} v\left[\mathbf{x}_{c k}\right]$ we obtain the expectation values

\footnotetext{
${ }^{2}$ We assume in the present work that the longest relaxation time $\tau_{\alpha}$ of the system becomes arbitrarily large, i.e. especially $\Delta \tau \ll \tau_{\alpha}$. The $c$-dependence drops out for ergodic systems with finite $\tau_{\alpha}$ and $\Delta \tau \gg \tau_{\alpha}$. See Sec. 2.2.9 of Ref. [6] for the $n_{\mathrm{c}^{-}}, n_{\mathrm{k}^{-}}$and $\Delta \tau$-dependences in the latter limit.
} 
for each configuration $c$. The expectation value over the complete $\left\{\mathbf{x}_{c k}\right\}$-ensemble is then given by the $c$-average $v \equiv \mathbf{E}^{c} v_{c}$. While $n_{\mathrm{c}}$ and $n_{\mathrm{k}}$ are assumed to be arbitrarily large, $n_{\mathrm{t}}$ is in general finite and for this reason $v_{c}(\Delta \tau)$ and $v(\Delta \tau)$ are apriori $\Delta \tau$-dependent as marked by the arguments. The relaxation processes may be characterized using functionals over $\mathbf{x}$ with a discrete time lag $t$ (with $\left.t=0, \ldots, n_{\mathrm{t}}-1\right)$ such as the "gliding average" 11

$$
c[\mathbf{x} ; t]=\frac{1}{n_{\mathrm{t}}-t} \sum_{i=1}^{n_{\mathrm{t}}-t} x_{i+t} x_{i} .
$$

We emphasize that the sum over $i$ is merely done to enhance the statistics since a stationary stochastic process does not change when shifted in time. As above we obtain by $k$-averaging the "autocorrelation function" (ACF) $c_{c}(\tau) \equiv \mathbf{E}^{k} c\left[\mathbf{x}_{c k} ; t\right]$ for a given configuration $c$ and in turn by $c$-averaging the ACF $c(\tau) \equiv \mathbf{E}^{c} c_{c}(\tau)$ of the entire $c k$ ensemble. It is useful to introduce the differences

$$
h_{c}(\tau) \equiv c_{c}(0)-c_{c}(\tau) \text { and } h(\tau)=c(0)-c(\tau) \text {. }
$$

A crucial point is that for stationary processes the sampling time dependence of $v_{c}(\Delta \tau)$ and $v(\Delta \tau)$ can be traced back to, respectively, $h_{c}(\tau)$ and $h(\tau)$. To state this compactly let us introduce the linear operator

$$
\begin{aligned}
\mathcal{L}_{\Delta \tau}[f] & \equiv \frac{2}{n_{\mathrm{t}}^{2}} \sum_{t=1}^{n_{\mathrm{t}}-t}\left(n_{\mathrm{t}}-t\right) f(t) \\
& \approx \frac{2}{\Delta \tau^{2}} \int_{0}^{\Delta \tau-\tau} \mathrm{d} \tau(\Delta \tau-\tau) f(\tau)
\end{aligned}
$$

where the first line states the discrete definition and the second line its continuum limit using that $\tau=t \delta \tau$ and $\Delta \tau=n_{\mathrm{t}} \delta \tau$. Note that for $a$ being a constant $\mathcal{L}_{\Delta \tau}[a]=a$ and this also holds if $f(\tau) \approx a$ for a finite but large time window 3,5 . Following the demonstration given, e.g., in Sec. 2.2 of Ref. [5], for the ergodic limit it can be seen that the stationarity assumption implies

$$
v_{c}(\Delta \tau)=\mathcal{L}_{\Delta \tau}\left[h_{c}\right]
$$

for each stationary configuration $c]^{3}$ Since $\left[\mathbf{E}^{c}, \mathcal{L}_{\Delta \tau}\right]=0$ we have similarly

$$
v(\Delta \tau)=\mathbf{E}^{c} v_{c}(\Delta \tau)=\mathcal{L}_{\Delta \tau}\left[\mathbf{E}^{c} h_{c}\right]=\mathcal{L}_{\Delta \tau}[h]
$$

for the $c k$-ensemble. The above relations Eq. (17) and Eq. (18) imply that $v_{c}(\Delta \tau)$ and $v(\Delta \tau)$ must vary strongly for sampling times $\Delta \tau$ corresponding to strong relaxation processes, i.e. for times $\tau \approx \Delta \tau$ where $h_{c}(\tau)$ and $h(\tau)$ strongly increase. On the other side $v_{c}(\Delta \tau)$ and $v(\Delta \tau)$ become constant in $\Delta \tau$-windows without or with few relaxation processes. The large time plateau values

$$
h_{c} \equiv \lim _{\tau \rightarrow \infty} h_{c}(\tau) \text { and } v_{c} \equiv \lim _{\Delta \tau \rightarrow \infty} v_{c}(\Delta \tau)
$$

\footnotetext{
${ }^{3}$ Eq. $\sqrt{17}$ is equivalent to $h_{c}(\tau)=\left(\tau^{2} v_{c}(\tau) / 2\right)^{\prime \prime}$ with the prime denoting a derivative with respect to $\tau$. This relation is closely related to the equivalence of the Green-Kubo formula and the Einstein relation for transport coefficients [5].
}

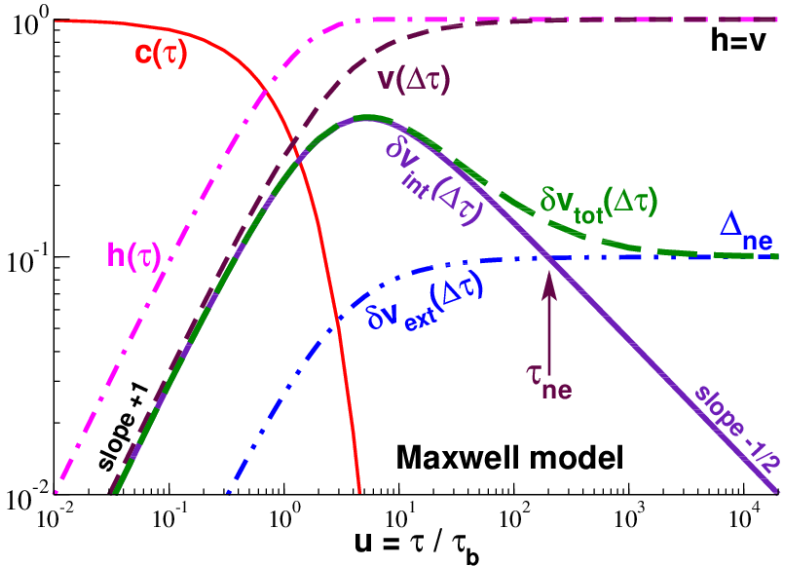

Fig. 1. Illustration of several properties for a Maxwell model The ACF is given by $c(\tau)=\exp (-u)$, with $u=\tau / \tau_{\mathrm{b}}$ being the reduced time and the non-ergodicity parameter $\Delta_{\text {ne }}=0.1$. We set $h=v=1$ and $\Delta_{\mathrm{ne}} / v=0.1 . c(\tau)$ is indicated by the thin solid line, $h(\tau)=c(0)-c(\tau)$ by the thin dot-dashed line, $v(\Delta \tau)$ determined according to Eq. 20 by the dashed line. $\delta v_{\text {ext }}(\Delta \tau)$ is obtained using Eq. 22$), \delta v_{\text {int }}(\Delta \tau)$ using Eq. (27) and $\delta v_{\text {tot }}(\Delta \tau)$ using Eq. (2).

(and similarly for $h$ and $v$ ) are relevant for times exceeding the basin relaxation time $\tau_{\mathrm{b}}$. It follows from Eq. (17) that $h_{c}=v_{c}$ and from Eq. (18) that $h=v$.

We illustrate various points made above by means of a Maxwell (Debye) model [5, 13, 18, i.e. we assume a stochastic process with one single exponentially decaying relaxation pathway. (More generally, response functions and correlation functions of many processes are successfully fitted by a linear superposition of a finite number or a distribution of such Maxwell modes [18.) This is presented in Fig. 1. The ACF $c(\tau)$ of the $c k$-ensemble is given by $c(\tau)=\exp (-u)$ as a function of the reduced time $u=\tau / \tau_{\mathrm{b}}$ using double logarithmic coordinates. It follows from Eq. (18) that 3.5

$$
v(\Delta \tau)=1-[\exp (-\Delta u)-1+\Delta u] 2 / \Delta u^{2}
$$

for $\Delta u=\Delta \tau / \tau_{\mathrm{b}}$. Let us for simplicity additionally assume that $h_{c}(\tau)$ is given by the product of a $c$-dependent constant and a $c$-independent time-dependence, i.e.

$$
h_{c}(\tau)=p_{c} h(\tau) \text { with } p_{c} \geq 0 \text { and } \mathbf{E}^{c} p_{c}=1 .
$$

With Eq. (17) and Eq. (18) this yields $v_{c}(\Delta \tau)=p_{c} v(\Delta \tau)$ Using Eq. (9) we have $\delta v_{\text {ext }}^{2}(\Delta \tau)=\left(\mathbf{V}^{c} p_{c}\right) v(\Delta \tau)^{2}$ which leads with Eq. (11) to

$$
\delta v_{\mathrm{ext}}(\Delta \tau) / \Delta_{\mathrm{ne}}=v(\Delta \tau) / v .
$$

As seen in Fig. 1 for $\Delta_{\text {ne }}=0.1, \delta v_{\text {ext }}(\Delta \tau)$ converges much faster than $\delta v_{\text {tot }}(\Delta \tau)$ to the common large- $\Delta \tau$ limit $\Delta_{\text {ne }}$.

\subsection{Gaussianity}

As further discussed in Sec. 3 many non-ergodic stochastic processes are in fact Gaussian within each meta-basin. 
Using exactly the same arguments put forward in Sec. 3.3 of Ref. [5] for ergodic Gaussian stochastic processes it can be shown using Wick's theorem, Eq. (44), that $\delta v_{c}(\Delta \tau)$ is then given by a functional $\delta v_{\mathrm{G}}\left[h_{c}\right]$ of the autocorrelation function $h_{c}(t)$. This functional is defined by [4,5]

$$
\begin{aligned}
\delta v_{\mathrm{G}}^{2}[f] & \equiv \frac{1}{2 n_{\mathrm{t}}^{4}} \sum_{i, j, k, l=1}^{n_{\mathrm{t}}} g_{i j k l}^{2} \text { with } \\
g_{i j k l} & \equiv\left(f_{i-j}+f_{k-l}\right)-\left(f_{i-l}+f_{j-k}\right)
\end{aligned}
$$

for any well-behaved function $f(t)$. Numerically better behaved reformulations of Eq. 23) are discussed in Ref. 5 . With $a$ and $b$ being real constants we have

$$
\delta v_{\mathrm{G}}[a]=0 \text { and } \delta v_{\mathrm{G}}[b(f-a)]=|b| \delta v_{\mathrm{G}}[f]
$$

and, hence, $\delta v_{\mathrm{G}}\left[h_{c}\right]=\delta v_{\mathrm{G}}\left[c_{c}\right]$. Equation (8) implies then

$$
\begin{aligned}
\delta v_{\mathrm{int}}^{2}(\Delta \tau) & =\mathbf{E}^{c} \delta v_{c}^{2}(\Delta \tau)=\mathbf{E}^{c} \delta v_{\mathrm{G}}^{2}\left[h_{c}\right] \\
& \approx \delta v_{\mathrm{G}}^{2}\left[\mathbf{E}^{c} h_{c}\right]=\delta v_{\mathrm{G}}^{2}[h]
\end{aligned}
$$

where the second line is an approximation replacing $h_{c}(t)$ by its $c$-average $h(t)$. This approximation is useful since $h_{c}(t)$ is not known in general, but rather $h(t)$ or $v(\Delta \tau)$.

Assuming again that Eq. (21) holds it is seen using the affinity relation Eq. 24) and Eq. 22, that

$$
\delta v_{\text {int }}^{2}(\Delta \tau)=(1+\epsilon) \delta v_{\mathrm{G}}^{2}[h] \text { with } \epsilon=\mathbf{V}^{c} p_{c} .
$$

Note that commonly $\epsilon \ll 1$, i.e. $\delta v_{\text {int }}(\Delta \tau) \approx \delta v_{\mathrm{G}}[h]$ in agreement with Eq. 26. As discussed in Sec. 3 and Sec. 4 $\epsilon \rightarrow 0$ for large systems with more or less independent microcells and the technical assumption Eq. (26) thus becomes increasingly rigorous. Equation 27) is also indicated in Fig. 1 (bold solid line). We take advantage of the fact that Eq. (23) can be solved analytically for the Maxwell model [5]. An important point is here that $\delta v_{\text {int }}(\Delta \tau)$ may quite generally become large, in fact of order of the expectation value $v(\Delta \tau)$, if $\Delta \tau$ corresponds to a relaxation time of the system. This is seen in Fig. 1 by the strong peak of $\delta v_{\text {int }}(\Delta \tau)$ at $u=\Delta \tau / \tau_{\mathrm{b}} \approx 6$. Note also that the total standard deviation $\delta v_{\text {tot }}(\Delta \tau)$ obtained from $\delta v_{\text {int }}(\Delta \tau)$ and $\delta v_{\text {ext }}(\Delta \tau)$ is given by $\delta v_{\text {tot }}(\Delta \tau) \approx \delta v_{\text {int }}(\Delta \tau)$ for $\Delta \tau \ll \tau_{\text {ne }}$ and by $\delta v_{\text {tot }}(\Delta \tau) \approx \delta v_{\text {ext }}(\Delta \tau) \approx \Delta_{\text {ne }}$ in the large- $\Delta \tau$ limit.

\section{System size effects}

\subsection{Phenomenological exponents}

Stochastic processes of many systems are to a good approximation Gaussian since $x_{t}=\mathbf{E}^{\mathbf{r}} x_{\mathbf{r} t}$ averages over many $\left(n_{\mathbf{r}} \gg 1\right)$ microscopic contributions $x_{\mathbf{r} t}$ and the central limit theorem applies [1. Albeit the $x_{\mathbf{r} t}$ may be spatially correlated (as discussed below) the fluctuations commonly decrease with $n_{\mathbf{r}}$. As a consequence, $h(\tau)$ and the related variances generally decrease with the system size. Assuming scale-free correlations one may write [5]

$$
\begin{array}{r}
h(\tau) \propto v(\Delta \tau) \propto \delta v_{\mathrm{G}}[h] \propto \delta v_{\text {int }}(\Delta \tau) \propto 1 / n_{\mathbf{r}}^{\hat{\gamma}_{\text {int }}} \\
\delta v_{\text {ext }}(\Delta \tau) \propto \Delta_{\text {ne }} \propto 1 / n_{\mathbf{r}}^{\hat{\gamma}_{\text {ext }}}
\end{array}
$$

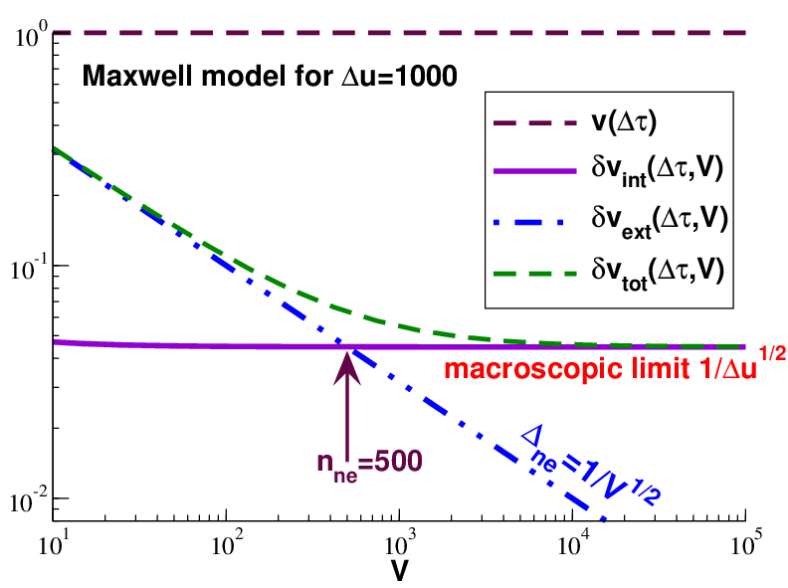

Fig. 2. System-size dependence of $v(\Delta \tau)$ and the corresponding standard deviations for the Maxwell model already presented in Fig. 2. It is supposed that $\Delta_{\text {ne }}=1 / \sqrt{V}$ and $\Delta u=\Delta \tau / \tau_{\mathrm{b}}=1000$. Even for such a huge sampling time it would be impossible to fit the correct exponent $\gamma_{\text {ext }}=1 / 2$ from the total standard deviation $\delta v_{\text {tot }}(\Delta \tau, V)$.

with $\hat{\gamma}_{\text {int }}$ and $\hat{\gamma}_{\text {ext }}$ being phenomenological exponents. That the asymptotic system-size effects for $h(\tau)$ and $v(\Delta \tau)$ are the same is due to Eq. (18). For Gaussian stochastic processes Eq. 26) implies the same exponent $\hat{\gamma}_{\text {int }}$ for $\delta v_{\mathrm{G}}[h]$ and $\delta v_{\text {int }}(\Delta \tau)$. As recalled in Ref. [6] $\hat{\gamma}_{\text {int }}=1$ and $\hat{\gamma}_{\text {ext }}=$ $3 / 2$ for strictly uncorrelated variables $x_{\mathbf{r}}$. The uncorrelated reference with $\hat{\gamma}_{\text {int }}=1$ is often included into the definition of the data entries by rescaling $x_{t}$ by a factor proportional to $\sqrt{n_{\mathbf{r}}}{ }^{4}$ Hence, $\hat{\gamma}_{\text {int }} \Rightarrow \gamma_{\text {int }} \equiv \hat{\gamma}_{\text {int }}-1$ and $\hat{\gamma}_{\text {ext }} \rightarrow \gamma_{\text {ext }} \equiv \hat{\gamma}_{\text {ext }}-1$ in the above relations, i.e. $\gamma_{\text {int }}=0$ and $\gamma_{\text {ext }}=1 / 2$ for rescaled uncorrelated variables $x_{t \mathbf{r}}$.

As an example we present in Fig. 2 the system-size dependence of the Maxwell model already discussed. We set $\Delta_{\text {ne }}=1 / \sqrt{V}$ and $V=n_{\mathbf{r}}$. Eq. $\sqrt{21}$ is again assumed and thus in turn also Eq. 22 and Eq. (27). We focus on one huge reduced sampling time $\Delta u=\Delta \tau / \tau_{\mathrm{b}}=1000$ where $v(\Delta \tau) \approx v=1$ and $\delta v_{\mathrm{G}}[h] \approx \sqrt{2 / \Delta u}\left[\underline{5} . \delta v_{\text {tot }}(\Delta \tau, V)\right.$ approaches the macroscopic limit $\delta v_{\text {int }}(\Delta \tau) \propto V^{0}$ for $V \gg$ $N_{\text {ne }} \approx \Delta u / 2$. Importantly, a crossover regime over at least two orders of magnitude is visible between both asymptotic limits. This implies that even if $\delta v_{\text {tot }}(\Delta \tau, V)$ is sampled with a huge constant $\Delta \tau$ an apparent exponent $\tilde{\gamma}_{\text {ext }}<$ $\gamma_{\text {ext }}=1 / 2$ may be measured due to the finite $\Delta \tau$. Exponents solely obtained from $\delta v_{\text {tot }}(\Delta \tau=$ const, $V)$ [16] may thus be misleading and should be considered with caution.

\subsection{Intensive thermodynamic fields}

We now assume that each $c$-trajectory is not only stationary and Gaussian but also at thermal equilibrium albeit under the constraints imposed to the meta-basin. We focus on instantaneous intensive thermodynamic variables $\sigma$

\footnotetext{
4 This rescaling is not only useful for strictly uncorrelated variables but also for general fluctuating thermodynamic fields as further discussed in Sec. 3.2
} 
(other than the temperature $T$ ) which are $d$-dimensional volume averages

$$
\sigma=\mathbf{E}^{\mathbf{r}} \sigma_{\mathbf{r}} \approx \frac{1}{V} \int \mathrm{d} \mathbf{r} \sigma_{\mathbf{r}}
$$

over fields $\sigma_{\mathbf{r}}$ of same dimension and $n_{\mathbf{r}}=V / \delta V$ being the number of microcells of volume $\delta V$. Following the rescaling convention made in Sec. 3.1 we use the rescaled variable $x \equiv \sqrt{V} \sigma$. As already stressed in Ref. [6], $\gamma_{\text {int }}=0$ must even hold for systems with long-range correlations if standard thermostatistics can be applied for each basin. To see this let us remind the reader that the large- $\Delta \tau$ limit $v_{c}$ of $v_{c}(\Delta \tau)$ is equivalent to the thermodynamically averaged variance of $x$ for the basin. Using the standard fluctuation-dissipation relation for the fluctuation of intensive thermodynamic variables [2,7,14] it is then seen that $v_{c}$ corresponds to a thermodynamic modulus of the $c$-basin which must be an intensive property, i.e. $\gamma_{\text {int }}=05^{5}$ Importantly, the same reasoning cannot be made for $\gamma_{\text {ext }}$, i.e. while $\gamma_{\text {int }}=0$ must hold $\gamma_{\text {ext }}=1 / 2$ may not for systems with long-range spatial correlations. The remainder of the paper illustrates this issue.

\subsection{Spatial correlations for $\tau_{\mathrm{b}} \ll \Delta \tau \ll \tau_{\alpha}$}

We have defined above the (generally $\Delta \tau$-depending) variance of a configuration $c$ by $v_{c}(\Delta \tau)=\mathbf{E}^{k} v\left[\mathbf{x}_{c k}\right]$ with $v[\mathbf{x}]=\mathbf{V}^{t} x_{t}$ being the $t$-averaged empirical variance of a given time series $\mathbf{x}$. We focus now on static properties obtained by $k$-averaging over asymptotically long $c$ trajectories and assuming $\tau_{\mathrm{b}} \ll \Delta \tau \ll \tau_{\alpha}$. In this limit not only the $\Delta \tau$-dependence of $v_{c}(\Delta \tau)$ drops out but due to the ergodicity within each basin the time $t$-average can be replaced by an ensemble $k$-average over the $x_{c k}$ of basin $c$. We thus lump $t$ - and $k$-indices together and the operator $\mathbf{E}^{k}$ replaces $\mathbf{E}^{k} \mathbf{E}^{t}$. $v_{c}$ is thus compactly redefined as

$$
v_{c} \equiv \mathbf{V}^{k} x_{c k}=\mathbf{E}^{k} x_{c k}^{2}-\left(\mathbf{E}^{k} x_{c k}\right)^{2}=V \mathbf{E}^{k} \delta \sigma_{c k}^{2}
$$

where we have used $\delta \sigma_{c k}=\sigma_{c k}-\mathbf{E}^{k} \sigma_{c k}$ in the last step. (The prefactor $V$ stems from the rescaling convention.) Using $\sigma_{c k}=\mathbf{E}^{\mathbf{r}} \sigma_{c k \mathbf{r}}$ and $\delta \sigma_{c k \mathbf{r}} \equiv \sigma_{c k \mathbf{r}}-\mathbf{E}^{k} \sigma_{c k \mathbf{r}}$ we write

$$
v_{c}=V \mathbf{E}^{k}\left(\mathbf{E}^{\mathbf{r}} \delta \sigma_{c k \mathbf{r}}\right)^{2}=V \mathbf{E}^{\mathbf{r}^{\prime}} \mathbf{E}^{\mathbf{r}^{\prime \prime}} \underline{\mathbf{E}^{k} \delta \sigma_{c k \mathbf{r}^{\prime}} \delta \sigma_{c k \mathbf{r}^{\prime \prime}}} .
$$

We define the pair (two-point) correlation function $C_{c}(\mathbf{r})$ as the average of the underlined term in Eq. 32 over all pairs $\mathbf{r}^{\prime}$ and $\mathbf{r}^{\prime \prime}=\mathbf{r}^{\prime}+\mathbf{r}$. Hence,

$$
v_{c}=V \mathbf{E}^{\mathbf{r}} C_{c}(\mathbf{r}) \approx \int \mathrm{d} \mathbf{r} C_{c}(\mathbf{r})
$$

\footnotetext{
${ }^{5}$ For the shear-stress fluctuations considered in Sec. $5 . v_{c}$ corresponds to the difference $\mu_{\mathrm{F}, c}=\mu_{\mathrm{A}, c}-\mu_{c}$ of the affine shear modulus $\mu_{\mathrm{A}, c}$ and the quasi-static shear modulus $\mu_{c} 2$, 3, 4, 5, 6] of the configuration $c$ for $\Delta \tau \rightarrow \infty$ with both $\mu_{\mathrm{A}, c}$ and $\mu_{c}$ being intensive properties.
}

with the first equation stating the discrete sum over all microcells and the second relation the corresponding integral for $\delta V \rightarrow 0$. Hence, $v=\mathbf{E}^{c} v_{c}=V \mathbf{E}^{\mathbf{r}} C(\mathbf{r})$ with $C(\mathbf{r}) \equiv \mathbf{E}^{c} C_{c}(\mathbf{r}){ }^{6}$

Similarly, $\Delta_{\text {ne }}^{2}=\delta v_{\text {ext }}^{2}=\mathbf{V}^{c} v_{c}$ may be rewritten exactly as an integral over the four-point correlation function $\mathbf{E}^{c}\left[\delta C_{c}\left(\mathbf{r}_{1}-\mathbf{r}_{2}\right) \delta C_{c}\left(\mathbf{r}_{3}-\mathbf{r}_{4}\right)\right]$ using $\delta C_{c}(\mathbf{r})=C_{c}(\mathbf{r})-$ $C(\mathbf{r})$. Unfortunately, without further approximations or physical assumption this does not yield a useful expression. One natural route to make progress is to identify a field allowing to express $\Delta_{\text {ne }}^{2}$ as in integral over a two-point correlation function. One possible field $s_{\mathbf{r}}$ is obtained by assuming that all isotropic and anisotropic contributions to the correlation function $C_{c}(\mathbf{r})$ of the fluctuating field $\sigma_{\mathbf{r}}$ rapidly decay on microscopic scales. We may thus approximate $v_{c}$, Eq. 32 , by the spatial average

$$
v_{c} \approx \mathbf{E}^{\mathbf{r}} s_{c \mathbf{r}}^{2} \text { with } s_{c \mathbf{r}} \equiv\left(\delta V \mathbf{E}^{k} \delta \sigma_{c k \mathbf{r}}^{2}\right)^{1 / 2}
$$

being the (rescaled) quenched standard deviation of $\left.\sigma_{c \mathbf{r}}\right]^{7}$ (The microscopic field may be renormalized for correlations of finite range.) As a consequence, $v=\mathbf{E}^{c} v_{c} \approx$ $\mathbf{E}^{c} \mathbf{E}^{\mathbf{r}} s_{c \mathbf{r}}^{2}$ and $\Delta_{\text {ne }}^{2}=\mathbf{V}^{c} v_{c} \approx \mathbf{V}^{c} \mathbf{E}^{\mathbf{r}} s_{c \mathbf{r}}^{2}$. Importantly, while the fluctuating field $\sigma_{c \mathbf{r}}$ is assumed to be short-ranged, this does not necessarily imply the same for the $k$-averaged field $s_{c \mathbf{r}}$. An alternative quenched field is discussed in Appendix B

\section{Simple models}

\subsection{Introduction}

We model for analytical and numerical simplicity the standard deviations $s_{c \mathbf{r}}$ by spatially correlated Gaussian fields $g_{c \mathbf{r}}$ (cf. Appendix A for details), i.e. $s_{c \mathbf{r}}=\left|g_{c \mathbf{r}}\right|$, and we focus on (static) moments and correlation functions of these fields. The approximation Eq. (34) is raised to a postulate, i.e. we assume that $v_{c}=\mathbf{E}^{\mathbf{r}} g_{c \mathbf{r}}^{2}$ and, hence,

$$
v=\mu_{2} \equiv \mathbf{E}^{c} \mathbf{E}^{\mathbf{r}} g_{c \mathbf{r}}^{2} \text { and } \Delta_{\text {ne }}^{2}=\Delta_{2}^{2} \equiv \mathbf{V}^{c} \mathbf{E}^{\mathbf{r}} g_{c \mathbf{r}}^{2}
$$

hold rigorously. More generally, we denote by $\mu_{l} \equiv \mathbf{E}^{c} \mathbf{E}^{\mathbf{r}} g_{c \mathbf{r}}^{l}$ the total average of the $l$ th moment and by $\Delta_{l}^{2} \equiv \mathbf{V}^{c} \mathbf{E}^{\mathbf{r}} g_{c \mathbf{r}}^{l}$ the corresponding variance. Using $\delta g_{c \mathbf{r}}^{l} \equiv g_{c \mathbf{r}}^{l}-\mu_{l}$ we get $\Delta_{l}^{2}=\mathbf{E}^{\mathbf{r}^{\prime}} \mathbf{E}^{\mathbf{r}^{\prime \prime}} \mathbf{E}^{c} \delta g_{c \mathbf{r}^{\prime}}^{l} \delta g_{c \mathbf{r}^{\prime \prime}}^{l}$. With $C_{l}(\mathbf{r})$ being the average of the underlined term over all pairs $\mathbf{r}^{\prime}$ and $\mathbf{r}^{\prime \prime}=\mathbf{r}^{\prime}+\mathbf{r}$ this implies $C_{l}(\mathbf{r}=0)=c_{l}=\mu_{2 l}-\mu_{l}^{2}$ and

$$
\Delta_{l}^{2}=\mathbf{E}^{\mathbf{r}} C_{l}(\mathbf{r})=\frac{1}{V} \int \mathrm{d} \mathbf{r} C_{l}(\mathbf{r})
$$

${ }^{6} v_{c} \geq 0$ sets a constraint on possible $C_{c}(\mathbf{r})$. Since $C_{c}(\mathbf{r})=$ $C_{c}(r, \underline{n})$ depends on the distance $r=\|\mathbf{r}\|$ and the direction $\underline{n}=\mathbf{r} / r$ one may write Eq. (33) as an $r$-integral of its isotropic average $C_{c}^{0}(r)$ over all $\underline{n}$. Due to the imposed (asymptotic) $V$ independence of $v_{c}$ for all basins (cf. Sec. 3.2 $C_{c}^{0}(r)$ and, hence, $C^{0}(r)=\mathbf{E}^{c} C_{c}^{0}(r)$ must decay more rapidly than $1 / r^{d}$.

7 A simple example is given by a magnetic spin system on a $d$-dimensional lattice subject to a strong external quenched magnetic field $H_{\mathrm{r}}$ and a weak, say Ising- or Heisenberg-type, coupling between neighboring spins 12,19 . 


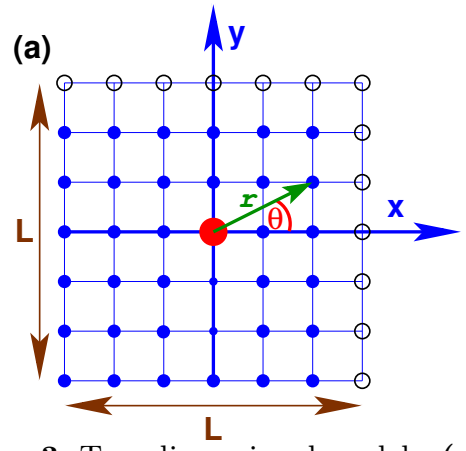

(b) model $\mathrm{D} L=100$

Fig. 3. Two-dimensional models: (a) sketch of periodic lattice for $L=6$ with filled circles indicating the cells of the principal box and open circles some periodic images. For microcells $\mathbf{r}$ of the principal box $C_{l}(\mathbf{r})$ is given by the distance $r$ and the angle $\theta$. (b) $C_{1}(\mathbf{r})$ for model D with $L=100, \alpha_{1}=0.5, \xi=\mu_{2}=1$, $\mu_{1}=0, d_{1}=0.5$.

Hence, $\Delta_{l}^{2}=c_{l} \delta V / V$ for spatially uncorrelated fields, i.e. for $C_{l}(\mathbf{r} \neq \mathbf{0})=0$. Importantly, for Gaussian fields $C_{l>1}(\mathbf{r})$ can be expressed in terms of $C_{1}(\mathbf{r})$ and the moments $\mu_{l}$. Specifically, as shown in Appendix A

$$
C_{2}(\mathbf{r})=2 C_{1}(\mathbf{r})^{2}+4 \mu_{1}^{2} C_{1}(\mathbf{r}) .
$$

Using Eq. (37) the r-average $\Delta_{2}^{2}$ over $C_{2}(\mathbf{r})$, Eq. (36), is thus set by $C_{1}(\mathbf{r})$ and $\mu_{1}$. For all model variants discussed below $C_{1}(\mathbf{0})=c_{1}=\mu_{2}-\mu_{1}^{2}$ holds, i.e. we need to specify additionally either $c_{1}$ or the moments $\mu_{1}$ and $\mu_{2}$.

\subsection{Model variants}

As sketched in panel (a) of Fig. 3 for the two-dimensional case, we use $d$-dimensional simple cubic lattices of unit lattice constant and linear dimension $L$ in all spatial directions, i.e. $n_{\mathbf{r}}=V=L^{d}$. Each of the $n_{\mathbf{r}}$ lattice sites corresponds to one microcell. As usual we use periodic boundary conditions 11 , i.e. $g_{\mathbf{r}}$ and the associated correlation functions $C_{l}(\mathbf{r})$ are $L$-periodic in all spatial directions. As indicated by filled circles in panel (a) of Fig. 3 we focus on the sites of the "principal simulation box" 11 characterized by the distance $r=\|\mathbf{r}\|$ from the origin (large filled circle) and the direction $\underline{n}=\mathbf{r} / r$.

We shall consider four model variants. "Model A" simply assumes that all microcells are uncorrelated, i.e. $C_{1}(r=$ $0)=c_{1}$ and $C_{1}(r>0)=0$. "Model B" assumes that the correlations decay exponentially

$$
C_{1}(\mathbf{r})=C_{1}(r)=c_{1} \exp (-r / \xi)
$$

with $\xi$ being the correlation length. Long-range correlations may appear in "model C" where

$$
C_{1}(\mathbf{r})=C_{1}(r)=c_{1}\left(1+(r / \xi)^{2}\right)^{-\alpha_{1} / 2}
$$

with $\xi$ being again a constant characterizing local physics and $\alpha_{1}>0$. The shifted power law is used to avoid a divergence at $r=0$ [19. Note that $C_{1}(r) \propto 1 / r^{\alpha_{1}}$ for $r \gg \xi$ and $r \gg 1$.
Up to now we have assumed that $C_{1}(\mathbf{r})$ only depends on the distance $r$ and not on the direction $\underline{n}$. Interestingly, even for isotropic systems $C_{l}(\mathbf{r})$ may depend on $\underline{n}$ if the stochastic variable $x(\tau)$ is only a component of a tensor and not a tensorial invariant. This is of relevance, e.g., for the shear-stress contribution of the stress tensor 20 , 21,22. Focusing on two-dimensional systems and using the angle $\theta$ shown in panel (a) of Fig. 3 our "model D" assumes $C_{1}(\mathbf{r}=\mathbf{0})=c_{1}$ and

$$
C_{1}(\mathbf{r})=\tilde{C}_{1}(r)\left[1-d_{1} \cos (4 \theta)\right] \text { for } r>0
$$

with $\tilde{C}_{1}(r)$ given by the power-law correlation of model C, Eq. (39). The period $\pi / 2$ is due to the fact that $C_{l}(x, y)$ is even, i.e. $C_{l}(x, y)=C_{l}(-x, y)=C_{l}(x,-y)=C_{l}(-x,-y)$, and the assumed equivalence of all spatial directions, i.e. $C_{l}(x, y)=C_{l}(y, x)$. This is known to hold especially for stress correlations in two-dimensional isotropic glasses 20 , 21,22. Interestingly, due to the discrete square lattice the "anisotropic" term in Eq. 40 may give finite contributions to the isotropic averages $C_{l}^{0}(r)$ over all possible $\theta$ for a given $r$ and (in turn to) the sums $\Delta_{l}^{2}$, Eq. (36), over all microcells. There are two reasons for this. For small $r$ the discrete lattice matters as may be seen by considering the cases $r=1$ or $r=2$. This effect becomes irrelevant for large $r \gg 1$ and $L$. More importantly, even for asymptotically large $L$ it matters for small exponents $\alpha_{1}$ that for $r \geq L / 2$ we only sample over microstates in the four corners of the lattice around the bisection lines $y= \pm x$ and a correspondingly reduced range of $\theta$ values. Since $\Delta_{l}^{2} \geq 0$, $d_{1}$ may not be too negative (depending on the other parameters). We focus on $d_{1}=0.5 . C_{1}(\mathbf{r})$ for $\alpha_{1}=0.5$, $\mu_{2}=\xi=1$ and $\mu_{1}=0$ is presented in panel (b) of Fig. 3

\section{3 $\Delta_{2}$ for Gaussian fields}

We present now $\Delta_{2}$ for the different models obtained equivalently by either numerically evaluating Eq. (37) or by explicitly first generating random fields (Appendix A) and averaging over $n_{\mathrm{c}}=10^{4}$ independent configurations. We focus first on the limit with $\mu_{1}=0$, i.e. $C_{2}(\mathbf{r})=2 C_{1}(\mathbf{r})^{2}$, and set $\mu_{2}=1$, i.e. $c_{1}=1$ and $c_{2}=2$.

If $C_{1}(\mathbf{r})$ and thus $C_{2}(\mathbf{r})$ are short-ranged, the systemsize must become rapidly irrelevant and, hence,

$$
\Delta_{2} \simeq 1 / V^{\gamma_{\text {ext }}} \text { with } \gamma_{\text {ext }}=1 / 2 .
$$

This behavior is shown in Fig. 4 for different one-dimensional $(d=1)$ systems. Since for model $\mathrm{A} C_{2}(\mathbf{0})=2$ and $C_{2}(\mathbf{r} \neq \mathbf{0})=0$, this implies $\Delta_{2}=\sqrt{2 / V}$ as indicated by the bold solid line. As one expects the data for model $B$ scales if traced as a function of the reduced volume $u=V / \xi^{d}$. Naturally, the scaling is not perfect for small $\xi$ due to the discrete lattice. For $u \ll 1$ we have $C_{2}(\mathbf{r}) \approx 2$ according to Eq. (37). As shown by the dashed horizontal line we thus have $\Delta_{2} \rightarrow \sqrt{2}$ for $u \ll 1$ while in the opposite limit $\Delta_{2} \simeq \sqrt{2 / u}$, as expected.

Long-range correlations may appear in model $\mathrm{C}$ as seen in Fig. 5. Since $\mu_{1}=0$ we have $\alpha_{2}=2 \alpha_{1}$ in the large- $r$ 


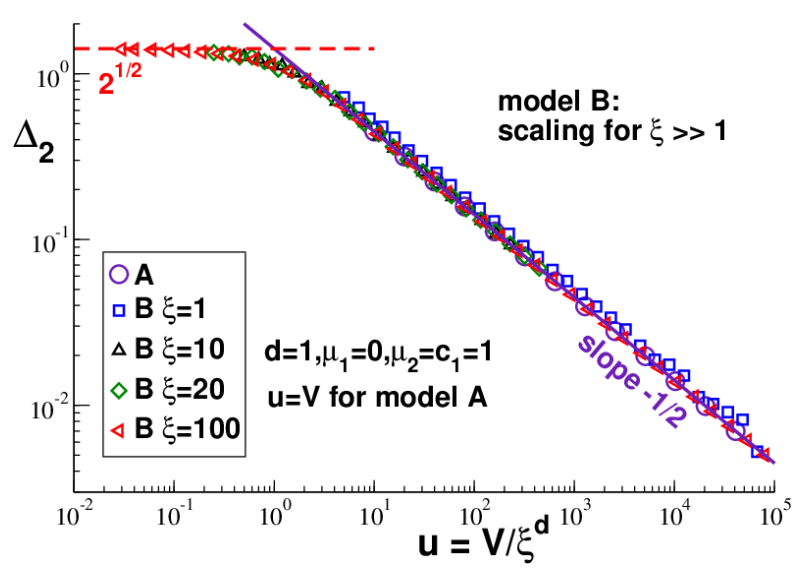

Fig. 4. $\Delta_{2}$ as a function of the (reduced) system size $u=V / \xi^{d}$ for models $\mathrm{A}$ and $\mathrm{B}$ for $d=1, \mu_{1}=0$ and $\mu_{2}=1$. While $\Delta_{2} \rightarrow \sqrt{2}$ for $u \ll 1$ (dashed horizontal line), $\Delta_{2} \simeq \sqrt{2 / u}$ for sufficiently large systems (bold solid line).

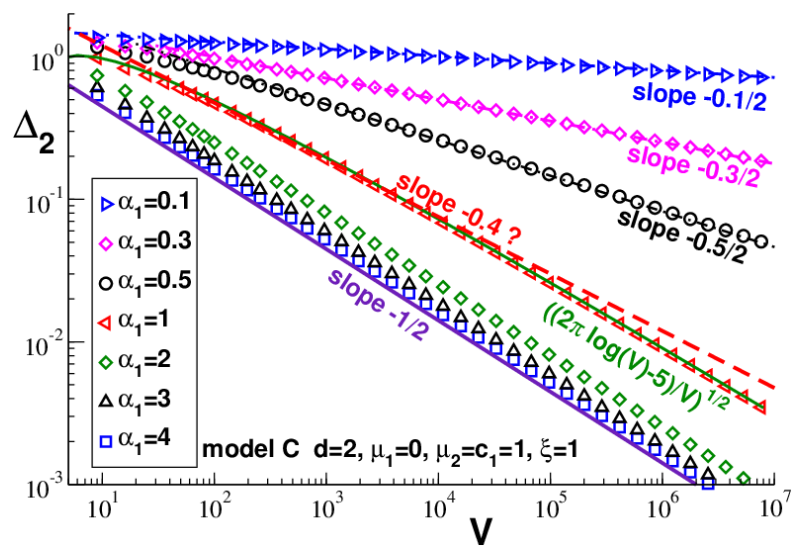

Fig. 5. $\Delta_{2}(V)$ for model C for $d=2, \mu_{1}=0, \mu_{2}=1, \xi=$ 1 and different power-low exponents $\alpha_{1}$ as indicated. While $\gamma_{\text {ext }}=1 / 2$ for $\alpha_{2}>d$ (bold solid line) and $\gamma_{\text {ext }}=\alpha_{1} / d$ for $\alpha_{d}<d$ (dash-dotted lines), an apparent exponent $\tilde{\gamma}_{\text {ext }} \approx 0.4$ (bold dashed lines) is observed for $\alpha_{2}=d$. The thin solid line indicates the logarithmic correction for $\alpha_{2} \rightarrow d$.

limit. Depending on the value of $\alpha_{2}$ and the spatial dimension $d$ it is readily seen from Eq. (36) that $\gamma_{\mathrm{ext}}=1 / 2$ for $\alpha_{2}>d$, while in the opposite limit

$$
\gamma_{\text {ext }}=\alpha_{2} / 2 d \text { for } \alpha_{2}<d
$$

Both relations are seen to hold in Fig. 5 for two-dimensional systems $(d=2)$. The cases with long-range correlations are emphasized by dash-dotted lines. For large $\alpha_{1}$ we see that $\Delta_{2}$ approaches the limit $\Delta_{2}=\sqrt{2 / V}$ (bold solid lines) of uncorrelated microcells (model A). Since

$$
\Delta_{2} \simeq(\log (V) / V)^{1 / 2} \text { for } \alpha_{2}=d
$$

we observe strong curvature for $\alpha_{1}=1$. Moreover, this limiting case is rather well fitted over at least two orders of magnitude by an apparent power law (bold dashed lines) with $\tilde{\gamma}_{\text {ext }} \approx 0.4$. (Similar results are obtained in other dimensions.) This demonstrates (if yet necessary) that such

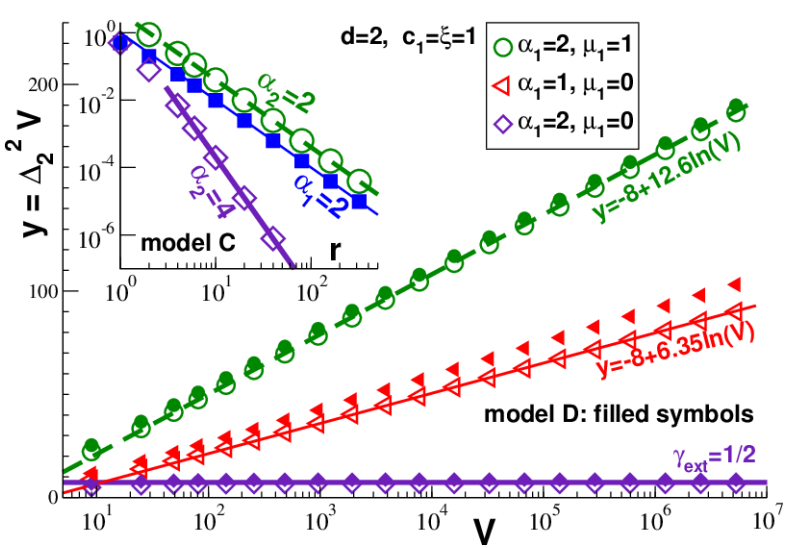

Fig. 6. Main panel: $y=\Delta_{2}^{2} V$ vs. $V$ for model C (open symbols) and model D (filled symbols). As shown by the bold horizontal line we have $\gamma_{\text {ext }}=1 / 2$ for $\alpha_{1}=2$ if $\mu_{1}=0$ while $y$ increases linearly for both models for $\mu_{1}=1$. Inset: Double-logarithmic representation of $C_{1}(\mathbf{r})$ (filled squares) and $C_{2}(\mathbf{r})$ (open symbols) for model $\mathrm{C}$ with $\alpha_{1}=2$ and $\mu_{1}=0$ (diamonds) and $\mu_{1}=1$ (circles). We have $C_{2}(\mathbf{r})=2 / r^{4}$ in the former case (bold solid line) and $C_{2}(\mathbf{r})=4 / r^{2}$ (dashed line) in the latter.

power-law fits should be treated with care. The thin solid line shows a logarithmic fit suggested by Eq. (43).

Up to now we have set $\mu_{1}=0$, i.e. $\alpha_{2}=2 \alpha_{1}$ for model $\mathrm{C}$ and model D. $\alpha_{1}=2$ thus implies $\alpha_{2}=4$, i.e. long-range correlations are irrelevant for $d=2$. This may be better seen using the half-logarithmic coordinates in the main panel of Fig. 6 where $y=\Delta_{2}^{2} V$ is plotted as a function of $V$. Indeed the data for $\mu_{1}=0$ and $\alpha_{1}=2$ (diamonds) are strictly horizontal (bold solid line) and logarithmic corrections only appear for $\alpha_{1}=1$ (triangles) ${ }^{8}$ Interestingly, the linear- $C_{1}(\mathbf{r})$-contribution in Eq. 377 is readily switched on using a finite $\mu_{1}$ and, as can be seen for model $\mathrm{C}$ in the inset of Fig. 6. $C_{2}(\mathbf{r}) \approx 4 \mu_{1} C_{1}(\mathbf{r})$ already for small $\mu_{1}$, i.e. $\alpha_{2} \approx \alpha_{1}$. As shown in the main panel, $\Delta_{2}$ reveals strong logarithmic behavior (circles).

\section{Mapping on shear-stress data}

It is tempting to tune the parameters of model C or D to fit the corresponding data obtained for shear-stresses in simulated model glasses $2,16,4,5,6$. We focus on systems formed by polydisperse Lennard-Jones (pLJ) particles in two dimensions. See Refs. 2,5] for a description of the Hamiltonian, the simulation method, the quench protocol and thermodynamic and structural properties. Boltzmann's constant and the average particle diameter are set to unity and Lennard-Jones units $[11$ are used. We impose a temperature $T=0.2$ - much smaller than the glass transition temperature $T_{\mathrm{g}} \approx 0.26[2,5]$ - and sample $n_{\mathrm{c}}=100$ independent configurations containing between $n=100$ and $n=40000$ particles. The number density is

\footnotetext{
8 The differences between models $\mathrm{C}$ and $\mathrm{D}$ for small $\alpha_{2}$ and large $L$ are due to the contributions of the anisotropic term of model D for discrete square lattices.
} 


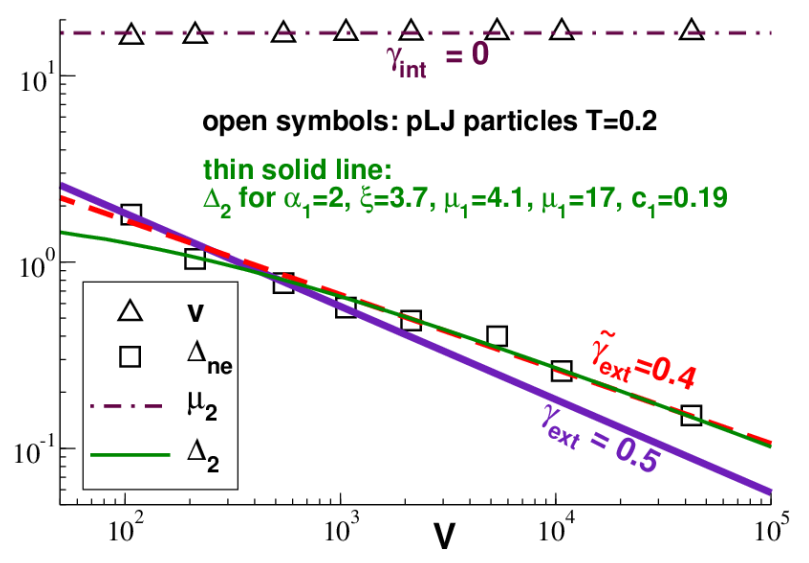

Fig. 7. Mapping of model $\mathrm{C}$ on data obtained from the shearstress fluctuations of MC simulations of pLJ particles in two dimensions (open symbols). As can be seen $v \approx 17$ is $V$ independent, i.e. $\gamma_{\text {int }}=0$. Imposing $\alpha_{1}=2$ and $\mu_{2}=v$ and fitting $\mu_{1}=4.1$ and $\xi=3.7$ yields $\Delta_{2}$ (thin solid line).

essentially system-size independent and of order unity, i.e. the particle number $n$ and the volume $V$ are numerically similar. The only observable relevant for the present work is the shear-stress contribution $\sigma$ to the (excess) stress tensor 11,5,6. Measurements are performed each MC step over a total sampling time $\Delta \tau_{\max }=10^{7}$. The stochastic process $x(\tau)$ is obtained as suggested by the convention made in Sec. 3.1 by rescaling $\sigma \Rightarrow x_{t} \equiv \sqrt{V / T} \sigma$. As described in Sec. 2.2 , we obtain from $v\left[\mathbf{x}_{c k}\right]$ the expectation value $v(\Delta \tau)$, the total variance $\delta v_{\text {tot }}^{2}(\Delta \tau)$ and its contributions $\delta v_{\text {int }}^{2}(\Delta \tau)$ and $\delta v_{\text {ext }}^{2}(\Delta \tau)$ and, finally, $\Delta_{\text {ne }}$ from the long-time limit of $\delta v_{\text {ext }}(\Delta \tau)$, Eq. (11). The $\Delta \tau$ - and $n_{\mathrm{k}}$-dependences are described in Ref. 6].

The data for $v\left(\Delta \tau=10^{6}\right) \approx v$ and $\Delta_{\text {ne }}$ are presented in Fig. 7. Since $v$ is the (rescaled) fluctuation of the shear stress, it is (essentially) $V$-independent, i.e. $\gamma_{\text {int }}=0$ in agreement with Sec. 3.2 As already emphasized elsewhere [5, 6], $\Delta_{\text {ne }}$ does not decay with an exponent $\gamma_{\text {ext }}=1 / 2$ (bold solid line) but with a weaker apparent exponent $\tilde{\gamma}_{\text {ext }} \approx 0.4$ (bold dashed line). This finding is qualitatively in agreement with the same exponent observed in Sec. 4 for either $\alpha_{1}=1$ and $\mu_{1}=0$ (Fig. 5) or $\alpha_{1}=2$ and $\mu_{1}=1$ (Fig. 6). Naturally, $\mu_{2}$ is set by the $V$-independent value of $v \approx 17$ as indicated by the dash-dotted horizontal line. Motivated by recent theoretical and numerical work [20, 21,22] we impose $\alpha_{1}=d=2$ and fit the remaining parameters $\xi$ and $\mu_{1}$ of model $\mathrm{C}$. This yields with $\xi \approx 3.7$ and $\mu_{1} \approx 4.1$ a nice fit (thin solid line) of $\Delta_{\text {ne }}$ for $n>100$. Similar values have been found for model $D$. The main limitation for a more critical test of the mapping is that $\Delta_{\text {ne }}$ is not known for larger system sizes.

\section{Conclusion}

Extending recent work [5, 6] the present study focused on the expectation value $v(\Delta \tau)$ and the standard deviations $\delta v_{\text {tot }}(\Delta \tau), \delta v_{\text {int }}(\Delta \tau)$ and $\delta v_{\text {ext }}(\Delta \tau)$ of the empirical vari- ance $v[\mathbf{x}]$ of time series $\mathbf{x}$, Eq. (1), of strictly non-ergodic stochastic processes recorded over a sampling time $\Delta \tau$.

Our first aim was to give an uncluttered summary (Sec. 2) of some useful notations (Sec. 2.1) and general relations important for the characterization of ensembles $\left\{\mathbf{x}_{c k}\right\}$ of such time series. At variance to ergodic processes the external standard deviation $\delta v_{\text {ext }}(\Delta \tau)$ becomes for non-ergodic systems constant, $\delta v_{\text {ext }}(\Delta \tau) \simeq \Delta_{\text {ne }}>0$, for large $\Delta \tau \gg \tau_{\mathrm{b}}$, and thus in turn so does also the total standard deviation $\delta v_{\text {tot }}(\Delta \tau)$ for $\Delta \tau \gg \tau_{\text {ne }}(V) \gg \tau_{\mathrm{b}}$, Eq. 12.

Our second aim was to emphasize by means of a simple analytically feasible example (Figs. 1 and 2 that it is therefore questionable to numerically determine the system-size exponent $\gamma_{\text {ext }}$ of $\Delta_{\text {ne }}(V)$ uniquely from the total standard deviation $\delta v_{\text {tot }}(\Delta \tau=$ const, $V)$. We argued that one should rather analyze the more rapidly converging $\delta v_{\text {ext }}(\Delta \tau, V)$ both with respect to $\Delta \tau$ and $V$.

Our third aim was to better understand the systemsize dependence of the static properties $v$ and $\Delta_{\text {ne }}$ for $\Delta \tau \gg \tau_{\mathrm{b}}$ in systems with correlated microcells (Sec. 3). We have thus investigated in Sec. 4 simple models where the (unaveraged) fluctuating microscopic contributions $\sigma_{\mathbf{r}}$ are essentially decorrelated but their (rescaled) $k$-averaged standard deviation $s_{\mathbf{r}}$ may not. For simplicity these frozen fields $s_{\mathbf{r}}=\left|g_{\mathbf{r}}\right|$ were modelled by spatially correlated Gaussian fields $g_{\mathbf{r}}$ (Appendix A). $v$ and $\Delta_{\text {ne }}$ are given, respectively, by the moments $\mu_{2}$ and $\Delta_{2}$ of the $g_{\mathbf{r}}$-field, Eq. (35). We have thus expressed $\Delta_{\text {ne }}$ in terms of an effective twopoint correlation function $C_{2}(\mathbf{r})$, Eq. (36). For consistency with general intensive thermodynamic fields (Sec. 3.2), $\mu_{2}$ is set to be $V$-independent $\left(\gamma_{\text {int }}=0\right)$. As seen in Fig. 4 for models $\mathrm{A}$ and $\mathrm{B}$ and in Fig. 5 for model $\mathrm{C}$ with $\alpha_{2}>d$, $\Delta_{2} \propto 1 / V^{\gamma_{\text {ext }}}$ with $\gamma_{\text {ext }}=1 / 2$ only holds for sufficiently strongly decreasing spatial correlations. Logarithmic corrections become relevant for models $\mathrm{C}$ and $\mathrm{D}$ for $\alpha_{2} \rightarrow d$ where $\Delta_{2}$ may be fitted over two orders of magnitude by an apparent exponent $\tilde{\gamma}_{\text {ext }} \approx 0.4$ (Fig. 5). A similar alternative approach yielding numerically equivalent results is mentioned in Appendix B.

Our fourth aim was to point out (Sec. 5) that rather similar behavior is observed for shear-stress fluctuations in amorphous glasses [6]. By insisting on $\alpha_{1}=d=2 \approx \alpha_{2}$ and tuning the parameters $\mu_{1}$ and $\xi$ of models $\mathrm{C}$ or $\mathrm{D}$ it was possible to fit the data (Fig. 7). This finding suggests the observed apparent exponent $\tilde{\gamma}_{\text {ext }} \approx 0.4$ [5, 6, 16 to be due to marginally long-range correlations of quenched shear-stress fluctuations.

Obviously, this does not necessarily imply that other aspects of the stress correlations in these systems are captured by simple models based on the key postulate Eq. (35) and, especially, on the technical relation Eq. (37) assuming correlated Gaussian fields. To clarify this issue future work [17] will focus on the characterization of the spatial correlations of different quenched fields such as the "local covariance field" $v_{c r}=V \mathbf{E}^{k} \delta \sigma_{c k \mathbf{r}} \delta \sigma_{c k}$ (Appendix B) which may be constructed from the shear stress fields $\sigma_{c k \mathbf{r}}$ numerically obtained following Lemaître 20. 
For simplicity of the presentation we have assumed in the present work a diverging longest system relaxation time $\tau_{\alpha}$ albeit for real physical, biological or socio-economical systems $\tau_{\alpha}$ is generally finite. Importantly, $\delta v_{\text {ext }}(\Delta \tau)$ must vanish in the ergodic limit for $\Delta \tau \gg \tau_{\alpha}$. It is appropriate for systems with a sluggish glass-like dynamics to redefine $\Delta_{\text {ne }}$ as the intermediate plateau value of $\delta v_{\text {ext }}(\Delta \tau)$. Naturally, all the presented results hold as long as $\Delta \tau, \tau_{\mathrm{b}}$ and $\tau_{\text {ne }}$ are much smaller than $\tau_{\alpha}$. The complete description of the standard deviations $\delta v_{\text {tot }}\left(\Delta \tau, n_{\mathrm{c}}, n_{\mathrm{k}}\right)$, $\delta v_{\text {int }}\left(\Delta \tau, n_{\mathrm{c}}, n_{\mathrm{k}}\right)$ and $\delta v_{\text {ext }}\left(\Delta \tau, n_{\mathrm{c}}, n_{\mathrm{k}}\right)$ is more intricate. See Sec. 4.6 of Ref. [6] for some first results.

\section{Author contribution statement}

JB, ANS and JPW designed the project. GG, LK and JPW performed the simulations. JPW wrote the manuscript benefitting from contributions of all authors.

\section{Acknowledgments}

We acknowledge computational resources from the HPC cluster of the University of Strasbourg.

\section{A Correlation functions of Gaussian fields}

Let $y_{i}$ be a normal distributed random field of zero mean, i.e. $\left\langle y_{i}\right\rangle=\mu_{1}=0$, with $i$ standing for the discrete time or spatial position. $(\langle\ldots\rangle$ stands here for a $c$-average over $n_{\mathrm{c}} \rightarrow \infty$ independent configurations.) Wick's theorem 1 thus holds, i.e.

$$
\begin{aligned}
& \left\langle y_{i} y_{j} y_{k} y_{l}\right\rangle= \\
& \left\langle y_{i} y_{j}\right\rangle\left\langle y_{k} y_{l}\right\rangle+\left\langle y_{i} y_{k}\right\rangle\left\langle y_{j} y_{l}\right\rangle+\left\langle y_{i} y_{l}\right\rangle\left\langle y_{j} y_{k}\right\rangle .
\end{aligned}
$$

This implies in turn $\left\langle y_{i}^{2} y_{j}^{2}\right\rangle-\left\langle y_{i}^{2}\right\rangle\left\langle y_{j}^{2}\right\rangle=2\left\langle y_{i} y_{j}\right\rangle^{2}$. With the indices corresponding to spatial positions and assuming translational invariance this shows that $C_{2}(\mathbf{r})=2 C_{1}(\mathbf{r})^{2}$ for $\mu_{1}=0$. If we consider instead the field $g_{i}=y_{i}+\mu_{1}$ with finite first moment $\mu_{1}=\left\langle g_{i}\right\rangle, C_{1}(\mathbf{r})$ remains unchanged while $C_{2}(\mathbf{r})$ does not. By substituting $y_{i}=g_{i}-\mu_{1}$, expanding $C_{2}(\mathbf{r})$ and using the invariance of $C_{1}(\mathbf{r})$ it is seen that more generally Eq. (37) holds 9

Random Gaussian fields $g_{\mathbf{r}}$ corresponding to the models of Sec. 4.2 have been explicitly generated numerically and we have measured the correlation functions $C_{l}(\mathbf{r}=$ $\mathbf{r}^{\prime \prime}-\mathbf{r}^{\prime}$ ) by averaging (consistently with the periodic boundary conditions) over all pairs of cells $\mathbf{r}^{\prime}$ and $\mathbf{r}^{\prime \prime}$ and the $n_{\mathrm{c}}$ independent configurations. Model A is trivially obtained by generating for each configuration $n_{\mathbf{r}}$ uncorrelated normal-distributed random numbers $\zeta_{\mathbf{r}}$ of zero mean and unit variance and setting $g_{\mathbf{r}}=\mu_{1}+a_{0} \zeta_{\mathbf{r}}$ with $c_{1}=a_{0}^{2}$. Spatially correlated random numbers $y_{\mathbf{r}}=g_{\mathbf{r}}-\mu_{1}$ are obtained by setting $y_{\mathbf{r}^{\prime}}=\sum_{\mathbf{r}^{\prime \prime}} a_{\mathbf{r}^{\prime} \mathbf{r}^{\prime \prime}} \zeta_{\mathbf{r}^{\prime}}$ where the "response"

\footnotetext{
${ }^{9}$ It is used here that $\left\langle s_{i}^{2} s_{j}\right\rangle-\left\langle s_{i}^{2}\right\rangle\left\langle s_{j}\right\rangle=2 \mu_{1} C_{1}(\mathbf{r})$.
}

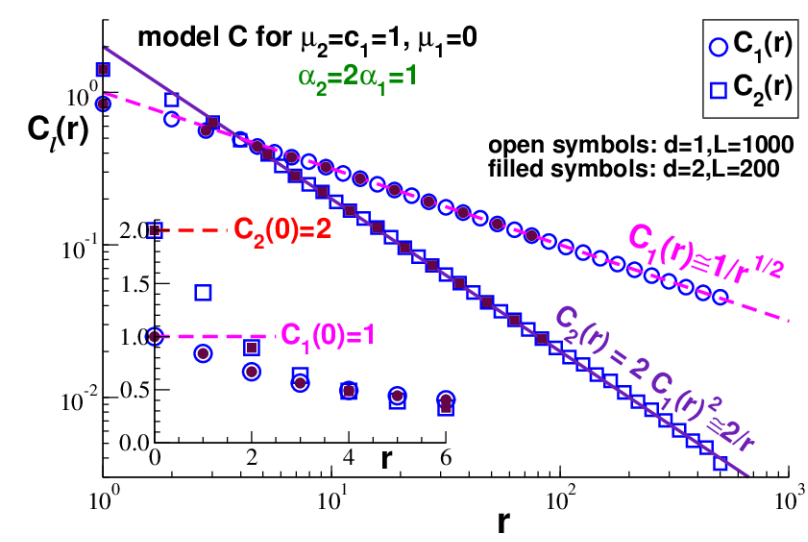

Fig. 8. $C_{1}(\mathbf{r})$ and $C_{2}(\mathbf{r})$ for model $\mathrm{C}$ with $\alpha_{1}=1 / 2, \mu_{1}=0$, $\mu_{2}=c_{1}=1$ and $\xi=1$. The open symbols have been obtained for $d=1$ and $L=1000$, the filled symbols for $d=2$ and $L=200$. Main: Double-logarithmic representation for logarithmically binned data. As emphasized by the solid line $\alpha_{2}=2 \alpha_{1}=1$. Inset: Linear representation for small $r$.

matrix $a_{\mathbf{r}^{\prime} \mathbf{r}^{\prime \prime}}$ is uniquely determined by the imposed correlation function $C_{1}(\mathbf{r})$ as shown below. Importantly, $g_{\mathbf{r}}=$ $y_{\mathbf{r}}+\mu_{1}$ is thus a linear superposition of Gaussian variables and therefore also Gaussian. 10 As a consequence Eq. 37. applies. Following a standard procedure 19 one way to obtain the $y_{\mathbf{r}}$-fields is to compute in turn the Fourier transforms $\zeta_{\mathbf{q}}=\mathcal{F}\left[\zeta_{\mathbf{r}}\right]$ and $C_{1}(\mathbf{q})=\mathcal{F}\left[C_{1}(\mathbf{r})\right]$, the product $y_{\mathbf{q}}=\sqrt{C_{1}(\mathbf{q})} \zeta_{\mathbf{q}}$, and finally the inverse Fourier transform $y_{\mathbf{r}}=\mathcal{F}^{-1}\left[y_{\mathbf{q}}\right]$ with $\mathcal{F}$ standing for the $d$-dimensional discrete Fourier transform and $\mathcal{F}^{-1}$ for its inverse. It is used here that $\zeta_{\mathbf{q}} \zeta_{-\mathbf{q}}=1$ and that $C_{1}(\mathbf{q})$ is real, even, positive and commensurate with the simulation box.

That the procedure works can be seen in Fig. 8 for model $\mathrm{C}$ in $d=1$ and $d=2$ (filled symbols). We have set $\alpha_{1}=1 / 2, \mu_{1}=0$ and $\mu_{2}=c_{1}=1$. The data for $C_{1}(\mathbf{r})$ and $C_{2}(\mathbf{r})$ are obtained by averaging over $n_{\mathrm{c}}=10^{4}$ independent configurations. Due to Eq. (37) we have $C_{2}(\mathbf{r}) \simeq$ $c_{2} / r^{\alpha_{2}}$ with $\alpha_{2}=2 \alpha_{1}=1$ as shown by the solid line in the main panel. The spatial dimension only plays a role for small and finite $r \approx 1$ as may be seen from the inset of Fig. 8. This leads to a weak $d$-dependence of integrals dominated by the lower integration bound.

\section{B Alternative quenched field}

An interesting alternative quenched field is given by the "local covariance" $v_{c \mathbf{r}} \equiv V \mathbf{E}^{k} \delta \sigma_{c k \mathbf{r}} \delta \sigma_{c k}$ between the local and total fluctuations $\delta \sigma_{c k \mathbf{r}}$ and $\delta \sigma_{c k}=\mathbf{E}^{\mathbf{r}} \delta \sigma_{c k \mathbf{r}}$. Note that $v_{c}=\mathbf{E}^{\mathbf{r}} v_{c \mathbf{r}}$ holds since $\mathbf{E}^{k}$ and $\mathbf{E}^{\mathbf{r}}$ commute. Importantly, for many physical systems $v_{c r}$ corresponds to a local modulus, e.g., the local stress-fluctuation contribution to an elastic modulus 15 . Using $\delta v_{c \mathbf{r}}=v_{c \mathbf{r}}-v$ we may

\footnotetext{
10 This implies that $g_{\mathbf{r}}$ may be negative even for large $\mu_{1}>0$ while the standard deviation $s_{\mathbf{r}}$ (cf. Sec. 3.3) of the microscopic field $\sigma_{\mathbf{r}}$ must be positive definite
} 
write quite generally without any additional assumption

$$
\Delta_{\text {ne }}^{2}=\mathbf{E}^{\mathbf{r}^{\prime}} \mathbf{E}^{\mathbf{r}^{\prime \prime}} \underline{\mathbf{E}}^{c} \delta v_{c \mathbf{r}^{\prime}} \delta v_{c \mathbf{r}^{\prime \prime}}=\mathbf{E}^{\mathbf{r}} C\left[v_{\mathbf{r}}\right](\mathbf{r})
$$

where $C\left[v_{\mathbf{r}}\right](\mathbf{r})$ stands for the average of the underlined term over all pairs of microcells $\mathbf{r}^{\prime}$ and $\mathbf{r}^{\prime \prime}=\mathbf{r}^{\prime}+\mathbf{r}{ }^{11}$ As a consequence, a slow $V$-decrease of $\Delta_{\text {ne }}$ with $\gamma_{\text {ext }}<$ $1 / 2$ must arise if $C\left[v_{\mathbf{r}}\right](\mathbf{r})$ is long-ranged. One may model the field $v_{\mathbf{r}}$ by means of a spatially correlated variable $g_{\mathbf{r}}$, i.e. using the notations of Sec. 4.1 we have $v=\mu_{1}$, $\Delta_{\text {ne }}=\Delta_{1}$ and the correlation function $C_{1}(\mathbf{r})$ corresponds to $C\left[v_{\mathbf{r}}\right](\mathbf{r})$. This yields a good alternative fit of the shearstress data discussed in Sec. 5 using model D with $\alpha_{1}=2$, $\mu_{1}=v=17.1, \mu_{2}=293$ and $\xi=3.3$. To discriminate between both modeling approaches a numerical comparison of correlation functions of different $k$-averaged quenched fields is warranted [17.

\section{References}

1. N.G. van Kampen, Stochastic processes in physics and chemistry (North-Holland, Amsterdam, 1992)

2. J.P. Wittmer, H. Xu, P. Polińska, F. Weysser, J. Baschnagel, J. Chem. Phys. 138, 12A533 (2013)

3. J.P. Wittmer, I. Kriuchevskyi, A. Cavallo, H. Xu, J. Baschnagel, Phys. Rev. E 93, 062611 (2016)

4. L. Klochko, J. Baschnagel, J.P. Wittmer, A.N. Semenov, J. Chem. Phys. 151, 054504 (2019)

5. G. George, L. Klochko, A. Semenov, J. Baschnagel, J.P. Wittmer, EPJE 44, 13 (2021)

6. G. George, L. Klochko, A.N. Semenov, J. Baschnagel, J.P. Wittmer, EPJE 44, 54 (2021)

7. M. Plischke, B. Bergersen, Equilibrium Statistical Physics (World Scientific, 1994)

8. A. Heuer, J.Phys.: Condens. Matter 20, 373101 (2008)

9. P. Charbonneau, J. Kurchan, G. Parisi, P. Urbani, F. Zamponi, Nature Commun. 5, 3725 (2014)

10. R. Metzler, J.H. Jeon, A. Cherstvy, Physical Chemistry Chemical Physics 16, 24128 (2014)

11. M.P. Allen, D.J. Tildesley, Computer Simulation of Liquids, 2nd Edition (Oxford University Press, Oxford, 2017)

12. D.P. Landau, K. Binder, A Guide to Monte Carlo Simulations in Statistical Physics (Cambridge University Press, Cambridge, 2000)

13. P.M. Chaikin, T.C. Lubensky, Principles of condensed matter physics (Cambridge University Press, 1995)

14. J.L. Lebowitz, J.K. Percus, L. Verlet, Phys. Rev. 153, 250 (1967)

15. J.F. Lutsko, J. Appl. Phys 65, 2991 (1989)

11 Being an $\mathbf{r}$-average over the pair correlation function of the covariance field, Eq. 45 is in fact a compact reformulation of the integral over the four-point correlation function mentioned in Sec. 3.3 While it is easier numerically to deal with pair correlation functions, it is in general challenging to get a phenomenological or complete analytical understanding of the scaling of $C\left[v_{\mathbf{r}}\right](\mathbf{r})$. This is, however, possible for shear stresses in viscoelastic fluids (including supercooled liquids and equilibrium amorphous systems) where $v_{\mathbf{r}}$ is related to the local elastic shear modulus [17.
16. I. Procaccia, C. Rainone, C.A.B.Z. Shor, M. Singh, Phys. Rev. E 93, 063003 (2016)

17. L. Klochko, A.N. Semenov, J. Baschnagel, J.P. Wittmer, in preparation (2021)

18. J.D. Ferry, Viscoelastic properties of polymers (John Wiley \& Sons, New York, 1980)

19. A.L. Barbabási, H. Stanley, Fractal Concepts in Surface Growth (Cambridge University Press, Cambridge, 1995)

20. A. Lemaître, Phys. Rev. Lett. 113, 245702 (2014)

21. M. Maier, A. Zippelius, M. Fuchs, Phys. Rev. Lett. 119, 265701 (2017)

22. L. Klochko, J. Baschnagel, J.P. Wittmer, A.N. Semenov, Soft Matter 14, 6835 (2018) 